\title{
Cerebrospinal fluid and serum uric acid levels in patients with multiple sclerosis
}

\author{
Irena Dujmovic ${ }^{1, *}$, Tatjana Pekmezovic ${ }^{1}$, \\ Radmila Obrenovic ${ }^{2}$, Aleksandra Nikolić ${ }^{3}$, \\ Mihailo Spasic ${ }^{3}$, Marija Mostarica Stojkovic ${ }^{4}$ \\ and Jelena Drulovic ${ }^{1}$ \\ ${ }^{1}$ Institute of Neurology, Clinical Center of Serbia, \\ Belgrade, Serbia \\ ${ }^{2}$ Institute of Medical Biochemistry, Clinical Center of \\ Serbia, Belgrade, Serbia \\ ${ }^{3}$ Institute for Biological Research "Sinisa \\ Stankovic", Belgrade, Serbia \\ ${ }^{4}$ Institute of Microbiology and Immunology, \\ Belgrade School of Medicine, Belgrade, Serbia
}

\begin{abstract}
Background: Peroxynitrite was hypothesized to be involved in the pathogenesis of multiple sclerosis (MS) through its various neurotoxic effects. Uric acid (UA) was shown to be a strong peroxynitrite scavenger.
\end{abstract}

Methods: We analyzed cerebrospinal fluid (CSF) and serum UA concentrations in $30 \mathrm{MS}$ patients and 20 controls with non-inflammatory neurological diseases (NIND) and correlated these findings with demographic and clinical characteristics of MS patients. Disease activity was assessed by brain magnetic resonance imaging (MRI) and the CSF/serum albumin quotient as an indicator of the state of blood-brainbarrier (BBB).

Results: Serum UA concentrations were found to be significantly lower in MS patients compared with controls $(p=0.019)$. CSF UA concentrations were lower in MS patients as compared to controls, as well as in patients with active MS (clinical and/or MRI activity) in comparison to patients with inactive MS or controls, but these differences were not statistically significant. Significant correlation was found between CSF and serum UA concentrations $(p=0.016)$ in MS patients, but not in controls; and between CSF UA concentrations and the CSF/serum albumin quotient in MS patients $(p=0.043)$, but not in controls.

Conclusions: Our results support the significance of $\mathrm{UA}$ in the pathogenesis of MS. Decreased serum UA concentrations in MS patients might be due to both intrinsically reduced antioxidant capacity and increased UA consumption in MS. CSF UA concentrations may not be a reliable marker of disease activity in MS since its concentration is dependent on leakage

\footnotetext{
*Corresponding author: Irena Dujmovic, Institute of Neurology, Clinical Center of Serbia, Dr Subotica 6, Belgrade 11000 , Serbia

Phone: +3816384 48 600, Fax: +381 112685 662, E-mail: irdujm@EUnet.rs

Received March 21, 2009; accepted April 21, 2009 previously published online June 4, 2009
}

of UA molecules from serum through the damaged BBB and the balance between consumption/production within the central nervous system (CNS). Clin Chem Lab Med 2009;47:848-53.

Keywords: cerebrospinal fluid (CSF); disease activity; multiple sclerosis (MS); serum; uric acid (UA).

\section{Introduction}

Multiple sclerosis (MS) is a chronic inflammatory and demyelinating disease of the central nervous system (CNS) (1). In the wide variety of immune-mediated mechanisms and molecules that could potentially cause CNS tissue damage in MS, many results point to the pathogenetic significance of nitric oxide (NO) (2-4). Several studies have shown that NO is produced in excess in the CNS (5-7) and NO metabolites are increased in body fluids of patients with MS (8-10). The toxicity of NO is significantly enhanced when it combines with superoxide to rapidly form the peroxynitrite anion (11). Peroxynitrite has been shown to be one of the most damaging free radicals and may exert its toxic effects directly, or by reducing the total antioxidant capacity through the number of mechanisms (12-14).

It has been shown that uric acid (UA) can scavenge not only peroxynitrite, but other free radicals $(15,16)$. $\mathrm{UA}$ is the final product of purine metabolism and accounts for $\sim 60 \%$ free radical scavenging activity in human blood (15). In a mouse model of MS, experimental autoimmune encephalomyelitis (EAE), the administration of exogenous UA was shown to prevent or suppress CNS tissue damage and clinical manifestations of the disease (17-19). Protective effects of UA on the blood-CNS barrier in EAE have been also demonstrated (20). Significantly lower serum UA concentrations were found in MS patients compared to controls in some studies (21-23), and low serum UA concentrations have been suggested to be a potential marker of disease activity in some MS patients (24).

In healthy subjects, CSF UA concentrations are 10 times lower compared with serum (25), and serum UA molecules can leak through the damaged blood-brainbarrier (BBB) (26). Available data on CSF UA concentrations in MS patients is scarce and controversial. Previous studies show CSF UA concentrations in MS patients to be either increased (27), the same as in control patients (28), or reduced in MS patients compared with controls (29). Only one study has investigated CSF UA concentrations in relation to disease activity (28). 
The aim of our study was to analyze CSF and serum UA concentrations in patients with MS and control patients, and to correlate these findings with demographic (gender, age), clinical (disease duration, disease course, neurological disability level, clinical disease activity) and paraclinical parameters (brain magnetic resonance imaging (MRI) activity, CSF/ serum albumin quotient).

\section{Materials and methods}

Blood and CSF samples were collected from 30 consecutive MS patients who were hospitalized at the Institute of Neurology, Clinical Center of Serbia, Belgrade, for diagnostic or therapeutic purposes. The diagnosis of MS was established according to the criteria of McDonald et al. (30). There were 21 females and nine males (M/F 1:2.5); mean age was 38.7 years (SD, 8.9; range 22-53 years), the mean duration of disease was 6.0 years (SD, 6.1; range $0.5-21$ years). Neurological disability was assessed using the expanded disability status scale (EDSS) (31). The median EDSS score in our patients was 4.5 (range, 1.0-8.0). Twenty-four patients had the relapsing-remitting (RR) form of the disease $(80 \%)$, three had secondary progressive (SP) $(10 \%)$ and three patients had primary progressive (PP) MS $(10 \%)$.

Fourteen patients in the RR patients group were in exacerbation, the remaining 10 were in remission. Exacerbation was defined according to the criteria of McDonald et al. (30). Collection of CSF and blood occurred during a two-week period following the onset of an exacerbation. Patients with progressive MS were considered to have clinically active disease when the EDSS score increased by at least one point from the previous year. Thus, two SP MS patients and three with PP disease were considered to have clinically active MS.

Additionally, all patients had brain MRI scans with gadopentetate dimeglumine (Gd-DTPA) injection performed the same day that blood and CSF were collected. MRI of the head was performed using a Siemens Magnetom (1.0 Tesla, Erlangen, Germany). The scanning protocol included T2weighted spin-echo images ( $T R=4000 \mathrm{~ms} ; \mathrm{TE}=90 \mathrm{~ms}$ ) and T1-weighted spin-echo images ( $T R=560 \mathrm{~ms} ; \mathrm{TE}=15 \mathrm{~ms}$ ), in the transverse plane, with a $5 \mathrm{~mm}$ slice thickness. Gd-DPTA was given intravenously at a dose of $0.1 \mathrm{mmol} / \mathrm{kg}$. Approximately $15 \mathrm{~min}$ after contrast injection, T1-weighted sequence was repeated. The same experienced neuroradiologist determined the presence of Gd-DTPA-enhancing lesions. The presence of at least one Gd-DTPA-enhancing lesion on the brain MRI served as a marker of MRI activity. Active lesions were found in 10 RRMS patients (one in remission and nine with exacerbation of RRMS).

According to the clinical and MRI criteria of disease activity, 20 patients were judged to have active MS (clinical and/ or MRI activity). Out of these 20 patients, 15 were with RRMS (14 patients with clinical exacerbation and one with clinically stable disease but with an active lesion on brain MRI). Two patients had SPMS and three had PPMS. The remaining 10 patients (nine with RR and one with SP disease) had inactive MS.

A group of 20 age- and sex-matched patients with other non-inflammatory neurological diseases (NIND): epilepsy $(n=6)$, lumbar disc herniation $(n=11)$, pseudotumor cerebri $(n=3)$, served as controls.

All MS patients and controls gave informed consent prior to participation in the study and the study was approved by the Local Ethics Committee. Excluded from the study were patients with chronic renal disease and diabetes mellitus, as well as those receiving acetylsalicylic acid, thiazide diuretics, steroids or other drugs which have been reported to modulate serum UA levels (32). None of the patients suffered from gout. Since diet can influence serum uric concentration based on its purine content, all patients received the same diet in the hospital for 7 days prior to blood/CSF sampling.

Lumbar puncture and blood collection were performed simultaneously in all patients after an overnight fast. Aliquots were immediately frozen at $-20^{\circ} \mathrm{C}$. UA concentrations in sera and CSF were determined using a commercially available enzymatic colorimetric assay according to the manufacturer's instructions (Randox-analyzer Monarch, Milan, Italy). In our institution, the normal range for serum UA is $140-400 \mu \mathrm{mol} / \mathrm{L}$ for females and $200-460 \mu \mathrm{mol} / \mathrm{L}$ for males.

Serum and the CSF albumin concentrations were determined using a commercially available nephelometric method according to the manufacturer's instructions (Behring, Marburg, Germany). The CSF/serum albumin quotient was used as an indicator of the integrity of the BBB.

The Mann-Whitney U-test was used for comparisons between groups. Spearman's rank correlation test was used to assess correlations.

\section{Results}

We analyzed CSF and serum UA concentrations in 30 MS patients and 20 NIND controls (Figure 1). Mean serum UA concentrations were significantly lower in MS patients $(267.2 \pm 70.2 \mu \mathrm{mol} / \mathrm{L}$; range, $110-407$ $\mu \mathrm{mol} / \mathrm{L})$, compared with controls $(294.5 \pm 55.13$ $\mu \mathrm{mol} / \mathrm{L}$; range, $199-400 \mu \mathrm{mol} / \mathrm{L})(p=0.019)$. Mean CSF UA concentrations were lower in MS patients (29.8 $\pm 12.3 \mu \mathrm{mol} / \mathrm{L}$; range, 2-55 $\mu \mathrm{mol} / \mathrm{L})$ compared with NIND controls $(35.2 \pm 14.0 \mu \mathrm{mol} / \mathrm{L}$; range, 19-72 $\mu \mathrm{mol} / \mathrm{L})$. However, this difference did not reach statistical significance $(p=0.082)$.

In patients with active MS, mean UA concentrations were lower in both the CSF $(28.5 \pm 13.7 \mu \mathrm{mol} / \mathrm{L}$; range, $2-53 \mu \mathrm{mol} / \mathrm{L})$ and serum $(262.65 \pm 75.3 \mu \mathrm{mol} / \mathrm{L}$; range, 110-407 $\mu \mathrm{mol} / \mathrm{L})$ compared with patients with inactive MS, in whom the mean value of CSF UA was $33.1 \pm 11.7 \mu \mathrm{mol} / \mathrm{L}$ (range, $12-55 \mu \mathrm{mol} / \mathrm{L}$ ) and the mean serum UA was $281.7 \pm 60.0 \mu \mathrm{mol} / \mathrm{L}$ (range,

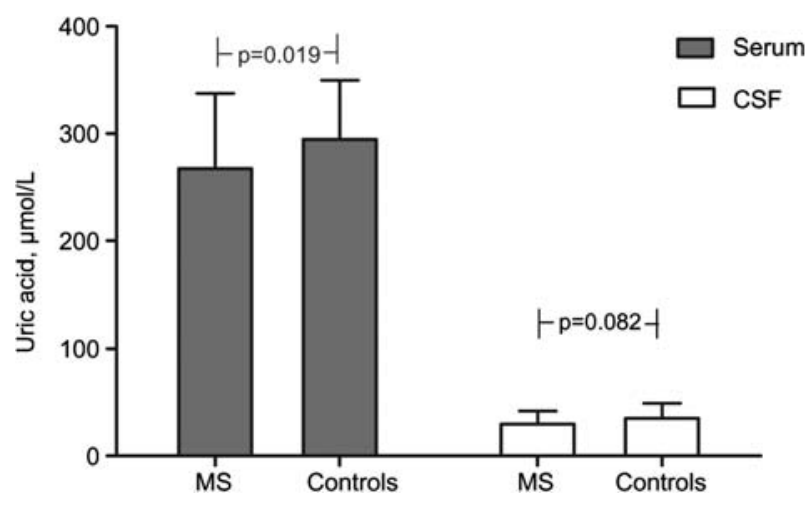

Figure 1 Concentration of uric acid in serum and cerebrospinal fluid from patients with multiple sclerosis, and controls with non-inflammatory neurological disorders.

MS, multiple sclerosis; CSF, cerebrospinal fluid. Bars represent means with standard deviation. 
200-373 $\mu \mathrm{mol} / \mathrm{L})$. However, this difference was not statistically significant $(p=0.271$, and $p=0.489$, respectively).

As shown in Figure 2, a trend toward a statistically significant difference between patients with active MS and controls was observed only in the mean CSF UA concentrations $(p=0.09)$ (Figure 2).

We found significant correlation between CSF and serum UA concentrations in patients with MS $(\rho=0.462, p=0.016)$. No correlation was found in NIND controls $(\rho=0.235, p=0.315)$.

CSF UA concentrations were highest in patients with the PP form of MS. Statistical comparisons regarding patients with PPMS $(n=3)$ and SPMS $(n=3)$ were not performed due to the small number of patients with progressive forms of the disease. CSF UA concentrations did not differ significantly between patients with RRMS and those with progressive forms of the disease (SPMS + PPMS) $(p=0.406)$ (Table 1). We did not find statistically significant differences between mean CSF UA concentrations in patients with RRMS vs. NIND controls $(p=0.186)$, and between patients with RRMS in relapse vs. patients with RRMS but in remission $(p=0.189)$.

No significant correlation was found between CSF UA concentrations and age of MS patients $(\rho=-0.20$,

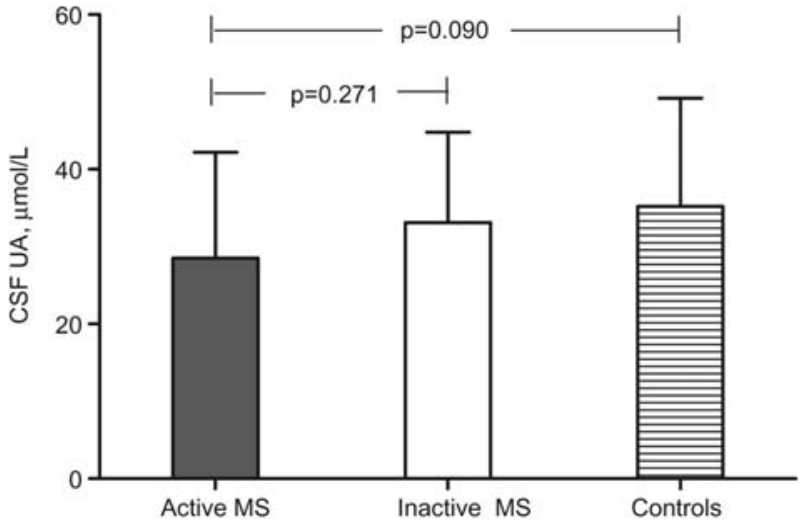

Figure 2 Comparison of the concentration of uric acid in cerebrospinal fluid from patients with active MS, inactive MS, and controls with non-inflammatory neurological disorders.

UA, uric acid; MS, multiple sclerosis; CSF, cerebrospinal fluid. Bars represent means with standard deviation. $p=0.916)$, duration of disease $(\rho=-0.030, p=0.873$ ) or degree of neurological disability as assessed by the EDSS score $(\rho=-0.282, p=0.131)$. However, significant correlation was found between CSF UA concentrations and female gender in MS patients $(\rho=-0.613$, $\mathrm{p}=0.0001$ ).

Significant correlation was also found between CSF UA concentrations and the CSF/serum albumin quotient (data not shown) in MS patients $(\rho=0.511$, $p=0.043)$, but not in controls $(\rho=-0.102, p=0.794)$.

\section{Discussion}

In our investigation, we found significantly lower serum UA concentrations in MS patients compared with NIND controls, and slightly lower, but not statistically significant, CSF UA concentrations in MS patients compared with NIND controls. Similarly, in patients with active MS, the mean UA concentration was lower both in the CSF and serum compared with patients with inactive MS. However, the difference between serum and CSF for the two MS patient groups was not statistically significant. We did observe a trend toward statistical significance for mean CSF UA concentrations in patients with active MS vs. controls. It is possible that this difference might be statistically significant if a larger patient group was studied.

In a study of $34 \mathrm{MS}$ patients and 20 controls with NIND, Stover et al. found 2-3-fold increases in CSF UA concentrations in MS patients compared with controls (27). Additionally, Becker et al. found normal UA concentrations in blood and CSF of 18 patients with MS (33). In a recent study by Zamani et al. (29), CSF and serum UA concentrations were measured in 12 patients with MS and 10 controls. Both CSF and serum UA concentrations were significantly less in MS patients compared with controls. However, in the above-mentioned studies, there are no data on clinical or brain MRI activity. A study by Kastenbauer et al. (28) investigated CSF UA concentrations in relation to disease activity. However, this study did not show any significant difference in serum and CSF UA concentrations between MS patients, with both active or inactive disease, and controls. The authors speculated that the patient selection for the

Table 1 Concentration of uric acid in cerebrospinal fluid of patients with various clinical phenotypes of multiple sclerosis.

\begin{tabular}{|c|c|c|c|c|}
\hline \multicolumn{2}{|l|}{ Patients } & \multicolumn{3}{|c|}{ Levels of uric acid in the cerebrospinal fluid, $\mu \mathrm{mol} / \mathrm{L}$} \\
\hline Disease course & No. & Mean $\pm S D$ & Median & Range \\
\hline $\mathrm{RRMS}^{\mathrm{a}}$ & 24 & $29.1 \pm 13.2$ & 28.5 & $2.0-55.0$ \\
\hline Relapse & 14 & $26.5 \pm 13.4$ & 24.0 & $2.0-53.0$ \\
\hline Remission & 10 & $33.4 \pm 12.4$ & 35.0 & $12.0-55.0$ \\
\hline SPMS $^{\mathrm{b}}$ & 3 & $23.7 \pm 9.2$ & 28.0 & $13.0-30.0$ \\
\hline Active & 2 & $20.5 \pm 10.6$ & 20.5 & $13.0-28.0$ \\
\hline Inactive & 1 & 30.0 & - & - \\
\hline $\mathrm{PPMS}^{\mathrm{c}}$ & 3 & $40.7 \pm 12.5$ & 41.0 & $28.0-53.0$ \\
\hline Active & 3 & $40.7 \pm 12.5$ & 41.0 & $28.0-53.0$ \\
\hline Inactive & 0 & - & - & - \\
\hline Total & 30 & $29.8 \pm 12.3$ & 28.5 & $2.0-55.0$ \\
\hline
\end{tabular}

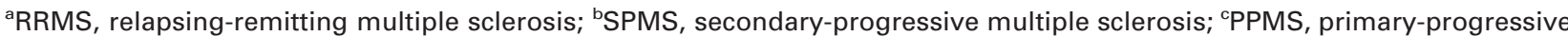
multiple sclerosis. 
control group could have influenced the results of this study (28).

Whether the reduction of UA concentrations in patients with active MS, as found in some studies $(21,29)$, is a cause or a consequence of disease activity remains unclear. It may be speculated that patients with active MS may have intrinsically reduced antioxidant capacity, which contributes to the tissue damage in MS. Epidemiological studies have found MS and gout, characterized by increased UA concentrations in serum, are mutually exclusive (17). Studies in twins showed that siblings with MS had lower serum UA concentrations compared with the healthy individual (22). Additionally, lower serum UA concentrations have been found in patients with optic neuritis (34), and in patients with other forms of the clinically isolated syndrome (CIS) (23). In accordance with these findings, we have previously demonstrated significantly lower mean serum UA concentrations in MS patients compared with NIND controls (21). Female gender correlated inversely with both serum UA concentrations in our previous study (21), and with CSF UA concentrations in this study. Thus, it could be presumed that the reduced antioxidant capacity, as assessed by the lower CSF UA concentrations in female patients with MS, could partially explain the predominance of females with MS. On the other hand, CNS inflammation in the active phase of the disease could lead to the consumption of UA and a reduction of the UA scavenger activity. In our previous study of 240 patients with MS, we found significantly lower serum UA concentrations in patients with clinical symptoms and/or MRI signs of disease activity (21).

Our results could support the potential significance of UA in the modulation of the immune inflammatory response in the CNS, as well as in the modulation of those processes that could damage the BBB in patients with MS. An animal model has shown that UA molecules in serum could leak through a damaged BBB (26). Our results may support this concept since we found significant correlation between CSF and serum UA concentrations in MS patients, but not in controls. Additionally, we have shown significant correlation between the CSF/serum albumin quotient, a marker for disruption of the BBB (35), and CSF UA concentrations in MS patients, but not in controls. Disruption of the BBB is one of the key events in the immunoinflammatory processes in MS (36). Therefore, we could assume that CSF UA concentrations depend partly on serum UA concentrations in patients with a damaged BBB, and partly on the balance between production and consumption within the CNS. This could partially explain the absence of a statistical significance in the CSF UA concentrations between patients with active and inactive MS in our study. However, the number of patients included in our study was rather small. We assume that statistically significant results could have been obtained in a larger group of MS patients.

The pathogenetic role of UA in MS also is supported by data showing the significant increase in serum UA concentrations in patients receiving interferon- $\beta$ or glatiramer-acetate $(37,38)$, which have been shown to reduce disease activity in MS (39). In addition, the administration of high-dose methylprednisolone for the treatment of MS relapse resulted in an increase in serum UA concentrations in the study by Toncev et al. (40).

The first steps toward the use of UA as a therapeutic agent in EAE were done by Hooper et al. who showed that treatment with UA inhibited the onset of acute EAE in mice (41). Additionally, treatment with UA was shown to promote long-term survival if treatment was begun after the onset of EAE symptoms (17). In addition, it also prevented disruption of the blood/CNS barrier and the invasion of inflammatory cells into the CNS in animals with EAE (18). In humans, oral administration of UA did not result in significant increases in serum UA concentrations (42). Thus, in a study by Spitsin et al. (43), administration of inosine, the precursor of UA, stopped the progression of MS in all 11 patients who received the drug; three patients showed some clinical improvement. Results of a phase II, double-blind, placebo-controlled study with the use of inosine in 30 RRMS and SPMS patients have not yet been published (http://clinicaltrials.gov/ct/show/NCT00067327). However, preliminary results indicate that manipulation of serum/CSF UA concentrations could hold promise in MS (24).

Our results further support the significance of UA in the pathogenesis of MS. Lowered serum UA concentrations in MS patients might be due to both intrinsically reduced antioxidant capacity and increased consumption of UA in MS. CSF UA concentrations may not be such a reliable marker of disease activity in MS because CSF concentrations are dependent on both leak of UA molecules into CSF from serum through the damaged $\mathrm{BBB}$ and the balance between consumption and production of UA within the CNS. However, our results may support the idea that treatment with UA precursors in patients with MS could have potential benefits for both stable and active disease. This may reduce the likelihood of disease reactivation and diminish the level of existing disease activity. Clinical studies with greater numbers of MS patients are necessary.

\section{Statement}

The authors have not accepted any funding or support from an organization that may in any way gain or lose financially from the results of this study or the conclusions of our review. The authors have not been employed by an organization that may in any way gain or lose financially from the results of this study and conclusions of our review. The authors disclose no other conflict of interests.

\section{References}

1. Lucchinetti CF, Bruck W, Rodriguez M, Lassmann H. Distinct patterns of multiple sclerosis pathology indicates heterogeneity on pathogenesis. Brain Pathol 1996;6: 259-74. 
2. Mitrovic B, Ignarro LJ, Montestruque S, Smoll A, Merrill JE. Nitric oxide as a potential pathological mechanism in demyelination: its differential effects on primary glial cells in vitro. Neuroscience 1994;61:575-85.

3. Mitrovic B, Parkinson J, Merrill JE. An in vitro model of oligodendrocyte destruction by nitric oxide and its relevance to multiple sclerosis. Methods 1996;10:501-13.

4. Garthwaite G, Goodwin DA, Batchelor AM, Leeming K, Garthwaite J. Nitric oxide toxicity in CNS white matter: an in vitro study using rat optic nerve. Neuroscience 2002;109:145-55

5. Bö L, Dawson TM, Wesselingh S, Mörk S, Choi S, Kong $\mathrm{PA}$, et al. Induction of nitric oxide synthase in demyelinating regions of multiple sclerosis brains. Ann Neurol 1994;36:778-86.

6. Bagasra O, Michaels FH, Zheng YM, Bobroski LE, Spitsin $\mathrm{SV}, \mathrm{Fu} Z \mathrm{~F}$, et al. Activation of the inducibile form of nitric oxide synthase in the brains of patients with multiple sclerosis. Proc Natl Acad Sci USA 1995;92:12041-5.

7. De Groot CJ, Ruuls SR, Theeuwes JW, Dijkstra CD, Van der Valk P. Immunocytochemical characterization of the expression of inducible and constitutive isoforms of nitric oxide synthase in demyelinating multiple sclerosis lesions. J Neuropath Exp Neur 1997;56:10-20.

8. Giovannoni G. Cerebrospinal fluid and serum nitric oxide metabolites in patients with multiple sclerosis. Mult Scler 1998;4:27-30.

9. Giovannoni G, Silver NC, O'Riordan J, Miller RF, Heales SJ, Land JM, et al. Increased urinary nitric oxide metabolites in patients with multiple sclerosis correlates with early and relapsing disease. Mult Scler 1999;5:335-41.

10. Drulović J, Dujmović I, Mesaros S, Samardzić T, Maksimović D, Stojsavljević N, et al. Raised cerebrospinal fluid nitrite and nitrate levels in patients with multiple sclerosis: no correlation with disease activity. Mult Scler 2001;7:19-22.

11. Beckman JS. Peroxynitrite versus hydroxyl radical: the role of nitric oxide in superoxide-dependent cerebral injury. Ann New J Acad Sci 1994;738:69-75.

12. Ischriopoulos $H$, Zhu L, Beckman JS. Peroxynitrite formation from macrophage-derived nitric oxide. Arch Biochem Biophys 1992;298:446-51.

13. Barker JE, Bolaños JP, Land JM, Clark JB, Heales SJ. Glutathione protects astrocytes from peroxynitrite-mediated mitochondrial damage: implications for neuronal/ astrocytic trafficking and neurodegeneration. Dev Neurosci 1996;18:391-6.

14. Pacher P, Beckman JS, Liaudet L. Nitric oxide and peroxynitrite in health and disease. Physiol Rev 2007; 87:315-424.

15. Ames BN, Cathcart R, Schwiers E, Hochstein P. Uric acid provides an antioxidant defense in humans against oxidant- and radical-caused aging and cancer: a hypothesis. Proc Natl Acad Sci USA 1981;78:6858-62.

16. Davies KJ, Sevanian A, Muakkassah-Kelly SF, Hochstein $P$. Uric acid-iron ion complexes. A new aspect of the antioxidant functions of uric acid. Biochem J 1986; 235:747-54

17. Hooper DC, Spitsin S, Kean RB, Champion JM, Dickson GM, Chaudhry I, et al. Uric acid, a natural peroxynitrite scavenger, in experimental allergic encephalomyelitis and multiple sclerosis. Proc Natl Acad Sci USA 1998; 95:675-80.

18. Hooper DC, Scott GS, Zborek A, Mikheeva T, Kean RB, Koprowski $\mathrm{H}$, et al. Uric acid, a peroxynitrite scavenger, inhibits CNS inflammation, blood-CNS barrier permeability changes and tissue damage in a mouse model of multiple sclerosis. FASEB J 2000;14:691-8.

19. Spitsin SV, Scott GS, Kean RB, Mikheeva T, Hooper DC. Protection of myelin basic protein immunized mice from free-radical mediated inflammatory cell invasion of the central nervous system by the natural peroxynitrite scavenger uric acid. Neurosci Lett 2000;292:137-41.

20. Kean RB, Spitsin SV, Mikheeva T, Scott GS, Hooper DC. The peroxynitrite scavenger uric acid prevents inflammatory cell invasion into the central nervous system in experimental allergic encephalomyelitis through maintenance of blood-central nervous system barrier integrity. J Immunol 2000;165:6511-8.

21. Drulović J, Dujmović I, Stojsavljević N, Mesaros S, Andjelković S, Miljković D, et al. Uric acid levels in sera from patients with multiple sclerosis. J Neurol 2001;248: 121-6.

22. Spitsin SV, Hooper DC, Mikheeva T, Koprowski H. Uric acid levels in patients with multiple sclerosis: analysis in mono- and dizygotic twins. Mult Scler 2001;7:165-6.

23. Rentzos M, Nikolaou C, Anagnostouli M, Rombos A, Tsakanikas K, Economou M, et al. Serum uric acid and multiple sclerosis. Clin Neurol Neurosur 2006;108: 527-31.

24. Spitsin S, Koprowski H. Role of uric acid in multiple sclerosis. Curr Top Microbiol Immunol 2008;318:325-42.

25. Lahoda F, Athen D. Typing of uric acid level in cerebrospinal fluid in neurological and psychiatric diseases. Adv Exp Med Biol 1977;76B:256-8.

26. Scott GS, Hooper DC. The role of uric acid in protection against peroxynitrite-mediated pathology. Medical Hypotheses 2001;56:95-100.

27. Stover JF, Lowitzsch K, Kempski OS. Cerebrospinal fluid hypoxanthine, xanthine and uric acid levels may reflect glutamate-mediated excitotoxicity in different neurological diseases. Neurosci Lett 1997;238:25-8.

28. Kastenbauer S, Kieseier BC, Becker BF. No evidence of increased oxidative degradation of urate to allantoin in the CSF and serum of patients with multiple sclerosis. J Neurol 2005;252:611-2.

29. Zamani A, Rezaei A, Khaeir F, Hooper DC. Serum and cerebrospinal fluid uric acid levels in multiple sclerosis patients. Clin Neurol Neurosur 2008;110:642-3.

30. McDonald WI, Compston A, Edan G, Goodkin D, Hartung $\mathrm{HP}$, Lublin FD, et al. Recommended diagnostic criteria for multiple sclerosis: guidelines from the International Panel on the diagnosis of multiple sclerosis. Ann Neurol 2001;50:121-7.

31. Kurtzke JF. Rating neurological impairment in multiple sclerosis: an expanded disability status scale (EDSS). Neurology 1983;33:1444-52.

32. Wortmann RL. Gout and other disorders of purine metabolism. In: Fauci SA, Braunwald E, Isselbacher JK, et al., editors. Harrison's principles of internal medicine. New York: McGraw-Hill, 1998;2158-66.

33. Becker BF, Kastenbauer S, Ködel U, KiesI D, Pfister HW. Urate oxidation in CSF and blood of patients with inflammatory disorders of the nervous system. Nucleosides Nucleotides Nucleic Acids 2004;23:1201-4.

34. Knapp CM, Constantinescu CS, Tan JH, McLean R, Cherryman GR, Gottlob I. Serum uric acid levels in optic neuritis. Mult Scler 2004;10:278-80.

35. Link $H$, Huang YM. Oligoclonal bands in multiple sclerosis cerebrospinal fluid: an update on methodology and clinical usefulness. J Neuroimmunol 2006;180:17-28.

36. Zlokovic BV. The blood-brain barrier in health and chronic neurodegenerative disorders. Neuron 2008;57:178201.

37. Guerrero AL, Martín-Polo J, Laherrán E, Gutiérrez F, Iglesias F, Tejero MA, et al. Variation of serum uric acid levels in multiple sclerosis during relapses and immunomodulatory treatment. Eur J Neurol 2008;15:394-7.

38. Constantinescu CS, Freitag P, Kappos L. Increase in serum levels of uric acid, an endogenous antioxidant, under treatment with glatiramer acetate for multiple sclerosis. Mult Scler 2000;6:378-81. 
39. Menge T, Weber MS, Hemmer B, Kieseier BC, von Büdingen $\mathrm{HC}$, Warnke $C$, et al. Disease-modifying agents for multiple sclerosis: recent advances and future prospects. Drugs 2008;68:2445-68.

40. Toncev G, Milicic B, Toncev S, Samardzic G. High-dose methylprednisolone therapy in multiple sclerosis increases serum uric acid levels. Clin Chem Lab Med 2002;40:505-8.

41. Hooper DC, Bagasra O, Marini JC, Zborek A, Ohnishi ST, Kean $R$, et al. Prevention of experimental allergic encephalomyelitis by targeting nitric oxide and peroxy- nitrite: implications for the treatment of multiple sclerosis. Proc Natl Acad Sci USA 1997;94:2528-33.

42. Koprowski H, Spitsin SV, Hooper DC. Prospects for the treatment of multiple sclerosis by raising serum levels of uric acid, a scavenger of peroxynitrite. Ann Neurol 2001;49:139.

43. Spitsin S, Hooper DC, Leist T, Streletz LJ, Mikheeva T, Koprowski $\mathrm{H}$. Inactivation of peroxynitrite in multiple sclerosis patients after oral administration of inosine may suggest possible approaches to therapy of the disease. Mult Scler 2001;7:313-9. 